Dermatologische Zeitschrift. 1913;20:I-VI

\title{
Contents, Vol. 20,1913
}

\section{Inhalts -Verzeichnis.}

S $\beta 1 t \beta$

Original-Arbeiten.

Bachrach, B., Kasuistischer Beitrag zur Kenntnis des

Lichen nitidus 189

Bettmann, S.. Spontane Rückbildung eines Naevus

vuerrcosus unius lateris $\quad 473$

Caesar, Viktor, Sieben Fälle von cerebralen Erkran-

kungen nach Salvarsanbehandlung 569

Demjanowitsch, M., Über eine seltene Form von

sekundärer Lues. (Syphilis cutanea verrucosa.) (Hierzu

Taf. IV.) $\quad 1 / 8 \quad 212$

Dreyer, A., Zur Klinik der Blasensyphilis . . 477, 591 Dudumi, V., Beitrag zur Kenntnis des Eczema marginatum

(Hebra) oder der Epidermophytia inguinalis (Sabouraud) 1082 Frieboes, W., Zwei Fälle von

Phlebitis und Periphlebitis

syphilitica faciei. Ein klinischer und histologischer

Beitrag. (Hierzu Tafel I-III.) 125

Géber, Hans, Nitrogen- und Schwefelstoffwechsel-

Untersuchungen bei Psoriasis vulgaris $\quad 377$

Ha na wa , S., Über die Beziehungen der Reizbarkeit der

menschlichen und tierischen Haut zu ihrem Pigment -

gehalt . \&lt; 761

Hochstetter, B., Über eine seltene Anomalie des

Haarwechsels 316

Hoffmann, E., Über Neurodermitis chronica faciei.

(Lichen simplex chronicus faciei) 117

ø-, Einiges aus dem Leben Julius Bettingers. des Pfälzers

Anonymus $22 \ddot{\mathrm{u}}$

, Über den Nachweis von Syphilisspirochäten in der

Hirnrinde bei Dementia paralytica durch H. Noguchi 375

und Bruno Hochstetter, Über eine Rollhaar-

cyste des Menschen nebst Bemerkungen zum Schrot-

ausschlag des Schweines (Hierzu Taf. IX bis XI.) . . 857

- IV -

Seite

Kyrle, J., Über das Rhinophyma, eine histologische Studie; zngleich ein Beit rag zur Frage der postfötalen Talgdrüsen- und Haarneubildung. (Hierzu Taf. VI-VII) 665

, Zur Frage der postfötalen Talgdrüsenneubildungen. 
(Hierzu Taf. XII-XIII.) 890

Lesser, F., Zur Verfeinerung der Wassermannschen Re-

aktion und Vermeidung divergent er Result ate .... 193

Lewinski, J., Beitrag zur Tuberkuiose des Penis . 692

Lube, F., Über epileptiforme Anfälle nach Salvarsan .

$\mathrm{M}$ i e d r e i c h, Franz, Klinischer und tierexperimenteller

Beitrag zur Toxikologie des Salvarsans 393

Müllern-Aspegren, U., Beitrag zur Abortiv-

behandlung der Syphilis mittels Salvarsan 992

, Zwei Fälle vonReinfektion von Syphilis undSalvarsan-

behandlung 1078

Polland, R., Naevus linearis verrucosus 499

-- - - , Über seltene Formen von Lichen ruber planus und ihre

Behandlung 778

R i c h t e r, P., Welche Bedeutung hat der ,,mal franzoso"

in Italien in der ersten Hälfte des 15. Jahrhunderts für

die Ansicht vom amerikanischen Ursprung der Syphilis ? $34 \mathrm{R}$ u e t e , A., Ein Fall von

Perifolliculitis capitis abscedens

et suffodiens. (Hierzu Taf. XIV-XV.) 901

Scherber, G., Zur Klinik und Atiologie einiger am

weibliehen Genitale auftretender seltener Geschwürs-

formen 140

Schlasberg, H. J., Zur Frage der Heilbarkeit der

Gonorrhoe bei Prostituierten 953

Schmidt, Ferdinand, Über Reetalgonorrhoe bei

Prostituierten 1065

Schumacher, J., Über Gonargin, ein neues Vaccine-

präparat $\quad 400$

S p r i n z, O., Ulerythema sycosiforme Unna (Sycosis lupoïde

Brocq). (Hierzu Taf. XVI.) 967

S t ü m p k e, G., Boeck $1 / 8$ ches Sarkoid bei ausgedehnter All-

gemeintuberkulose 199

$\mathrm{S}$ u d h o ff , K., Anfänge der Syphilisbeobaehtung und Sy-

philisprophylaxe zu Frankfurt a. M. 1496-1502 ... 95

. ,,Amerikanischer Ursprung” und ,,mal franzoso" iim

die Mitte des 15. Jahrhunderts in Italien 325 

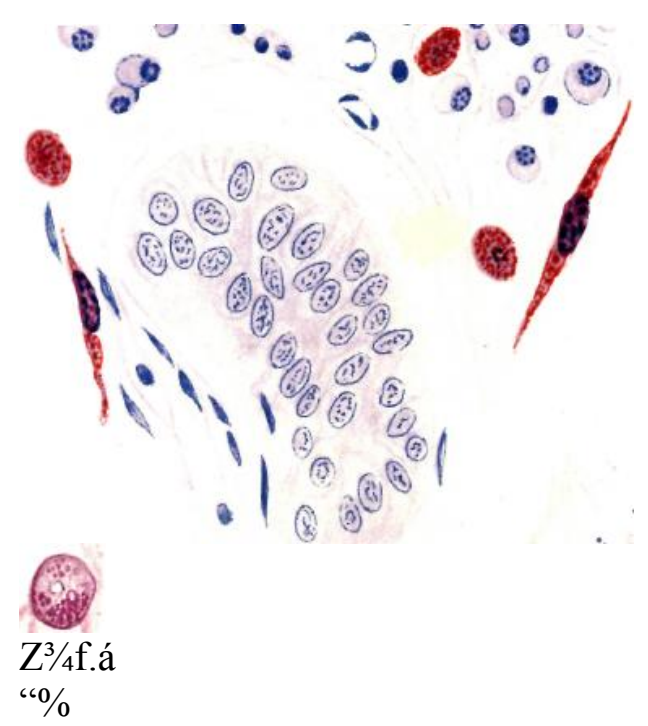

7/83/81. \&gt; : ·á $\mathrm{va}^{1 / 81} \mathbf{1} \mathbf{1}^{-} \cdot \ldots$

Dermatolorgissthe Zeitschrift, Bd. XI:

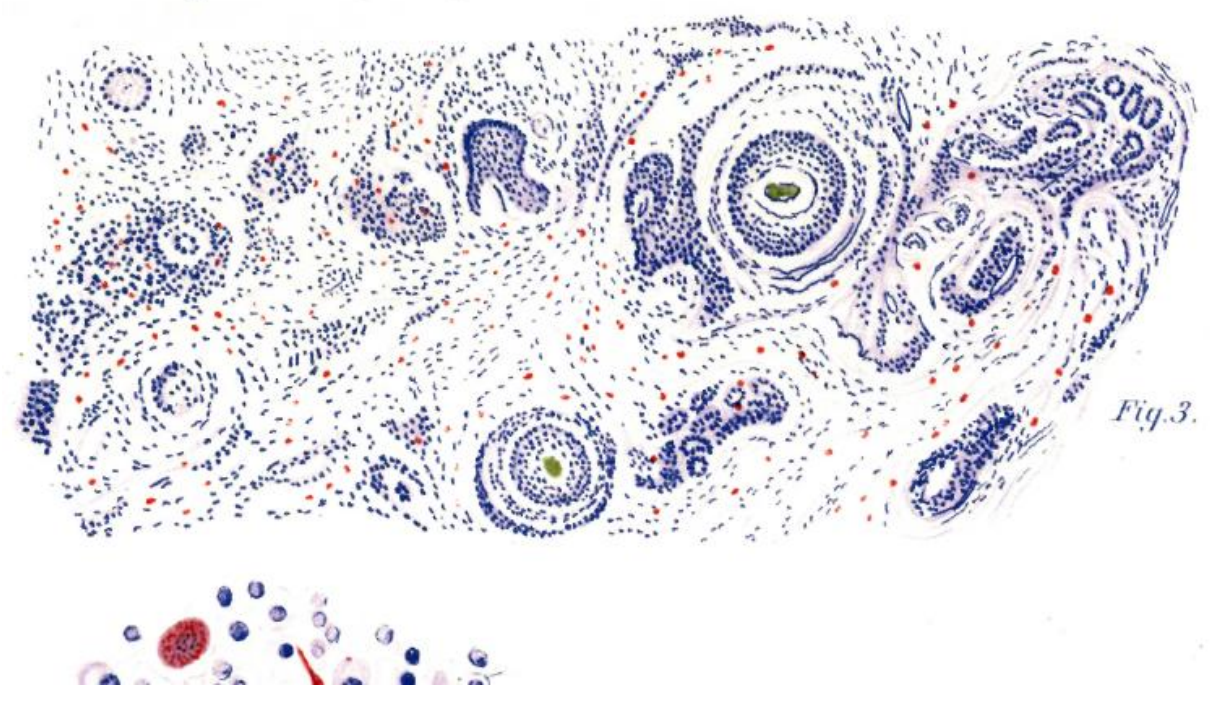

(0.

$\%$

$\& 1 t$

$\cdot 7 / 8 \mathrm{~s}$.

$\% \mathrm{E}$ 
$1 / 8 \Gamma-\cdot$

| $\psi$ rir\%oz.

- S. Karijer in, BerliræW $\beta$.

$-\mathrm{V}-$

Sßite Tomasczewski, E., Über die Todesfälle nach intra-

venösen Injektionen von Salvarsan und Neosalvarsan

283, 411 Toyama, J., Beitrag zur Kenntnis der Acanthosis

nigricans. (Hierzu Taf. VIII.) 785

Vignolo-Lutati, Karl, Beitrag zum Studium der

Sklerodermie der Zunge und der Mundschleimhaut . 682

Vörner, H., Urtica solitaria

Gesellschaftsberichte.

Bericht über den XVII. internationalen medizinischen Kongreß in London (Dermatologische

Sektion). VonPriv.-Doz. Dr. R. P o 11 a n d und Priv.-Doz. Dr. W. Frieboes 911

XI. Kongreß der Deutschen Dermatologischen Gesellschaft

in Wien am 19. und 20. September 1913. Nach Auto-

referaten zusammengestellt von DDr. G. Scherber

und $\mathrm{U} n \mathrm{~g}$ a $\mathrm{r}$ in Wien 998

82. Versammlung Deutscher Naturforscher und Arzte in

Wien. Nach Autoreferaten zusammengestellt von DDr.

G. Scherber und U $\mathrm{n} g$ a $\mathrm{r}$ in Wien 1011

II. Kongreß der .,Nordisk Dermatologisk Forening” zu

Stockholm vom 5.--7. Juni 1913. Von Dr. Poul

Haslund in Kopenhagen 1110

IV. Internationaler Kongreß für Physiotherapie vom 26.

bis 30. März 1913 (Charité). Von Dr. Arthur

Alexander in Charlottenburg 502

IX. Kongreß der Deutschen Röntgengesellschaft am 29. und

30. März in Berlin-Langenbeckhaus. Von Dr. Arthur

Alexander in Charlottenburg 515

Lupus-Ausschuß des Deutschen Zentral-Komitees zur Be-

kämplüng der Tuberkulose am 27. Oktober 1913 in

Berlin-Charité. Von Dr. Arthur Alexander in

Charlottenburg $\quad 1116$

Berliner Dermatologische Gesellschaft. Sitzungen am 12. November, 10. Dezember 1912, 14.

Januar, 11. Februar, 11. März, 20. Mai, 10. Juni und 8. Juli 1913 . .149,

223, 330, 440, 705, 796, 806, 1120

Moskauer Venerologisch - dermatologische Gesellschaft.

Sitzungen am 25. Oktober (7. November) 1912 und am

27. Januar (9. Februar) 1913 47, 346

$-\mathrm{VI}-$

Paris er Dermatologische Gesellschaft. Sitzungen am 7. November und 5. Dezember 1912, 6.

Februar 3. April, 8. Mai, 5. Juni und 3. Juli 1913 .... 163, 239,

623, 712, 827, 923 Wiener Dermatologische Gesellschaft. Sitzungen am 3D. Ok-tober, 13.

November, 4. Dezember 1912, 15. und 29. Januar, 12. und 26. Februar, 23. April, 7. und 
21. Mai und 4. Juni $1913 \quad 41,159$,

236, 336, 444, 631, 709, 817

Spirochäten bei Paralyse und Tabes 472

Periodische Literatur 48, 168, 243, 347, 448, 518, 637,

$715,829,926,1031,1136$

Buehanzeigen 89, 185, 279, 663, 1057, 1158

Tagesnaehrichten und Personalien 94, 188, 281, 376, 472,

$568,664,757,760,856,951,1064,1160$

Jonathan Hutchinson $\dagger$

Prof. Dr. Carl Koppf

760

Sachregister

94

Namenregister

1161

1174 